CRYSTALLOGRAPHIC COMMUNICATIONS

ISSN 2056-9890

Received 16 March 2018

Accepted 13 April 2018

Edited by $\mathrm{H}$. Stoeckli-Evans, University of Neuchâtel, Switzerland

Keywords: crystal structure; cyclohexane rings; tetrahydropyran rings; helical supramolecular chains; Hirshfeld surface analysis.

CCDC reference: 1837011

Supporting information: this article has supporting information at journals.iucr.org/e

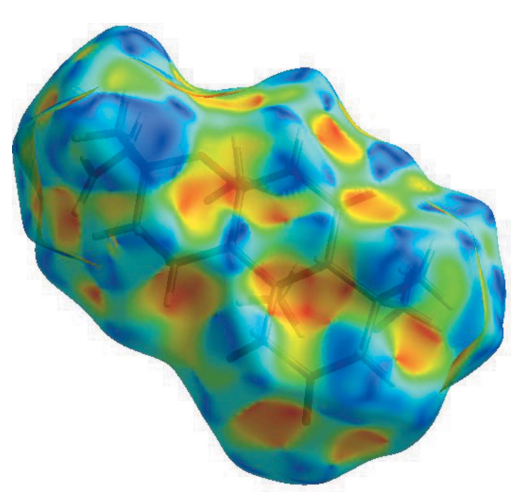

OPEN $\odot$ ACCESS

\section{Crystal structure and Hirshfeld surface analysis of 2-oxo-13-epi-manoyl oxide isolated from Sideritis perfoliata}

\author{
Ísmail Celik, ${ }^{a}$ Zeliha Atioğlu, ${ }^{\text {b }}$ Huseyin Aksit, ${ }^{\text {c }}$ Ibrahim Demirtas, ${ }^{\text {d }}$ Ramazan \\ Erenler ${ }^{\mathrm{e}}$ and Mehmet Akkurt ${ }^{\mathrm{f}_{*}}$
}

aDepartment of Physics, Faculty of Sciences, Cumhuriyet University, 58140 Sivas, Turkey, bilke Education and Health
Foundation, Cappadocia University, Cappadocia Vocational College, The Medical Imaging Techniques Program, 50420
Mustafapaşa, Ürgüp, Nevşehir, Turkey, 'Erzincan University, Faculty of Pharmacy, 24100 Erzincan, Turkey, ${ }^{\mathbf{d}}$ Department
of Chemistry, Faculty of Natural Sciences, Cankiri Karatekin University, 18100 Cankiri, Turkey, ${ }^{\mathbf{e}}$ Department of
Chemistry, Faculty of Arts and Sciences, Gaziosmanpasa University, 60240 Tokat, Turkey, and ' Department of Physics,
Faculty of Sciences, Erciyes University, 38039 Kayseri, Turkey. ${ }^{*}$ Correspondence e-mail: akkurt@erciyes.edu.tr

The title compound, $\mathrm{C}_{20} \mathrm{H}_{32} \mathrm{O}_{2}$ (systematic name: 3-ethenyl-3,4a,7,7,10a-pentamethyldodecahydro-9H-benzo[f]chromen-9-one), was isolated from Sideritis perfoliata. In the crystal, molecules pack in helical supramolecular chains along the $2_{1}$ screw axis running parallel to the $a$ axis, bound by $\mathrm{C}-\mathrm{H} \cdots \mathrm{O}$ hydrogen bonds. These chains are efficiently interlocked in the other two unit-cell directions via van der Waals interactions. Hirshfeld surface analysis shows that van der Waals interactions constitute the major contribution to the intermolecular interactions, with $\mathrm{H} \cdots \mathrm{H}$ contacts accounting for $86.0 \%$ of the surface.

\section{Chemical context}

The genus Sideritis belonging to the Lamiaceae family is represented by more than 150 species, distributed in tropical regions. Most of the species are found in the Mediterranean region. This genus is represented by 54 species in Turkey flora, 40 of which are endemic (Davis, 1982). Sideritis species have traditionally been used as herbal teas, flavouring agents and therapeutics (Danesi et al., 2013). Sideritis species include flavonoids, terpenes, iridoids, coumarins, lignanes and sterols that are responsible constituents for their pharmacological properties (González-Burgos et al., 2011). Sideritis species have been reported to exhibit considerable biological activities such as antioxidant (Demirtas et al., 2011), antiproliferative (Demirtas et al., 2009), and antimicrobial (Yiğit Hanoğlu et al., 2017) effects. The crystal structure of 2- $\beta$-hydroxymanoyl oxide isolated from Sideritis perfoliata has been reported on by our group (Çelik et al., 2016). Herein, we report on the crystal structure of 2-oxo-13-epi-manoyl oxide, also isolated from $S$. perfoliata.

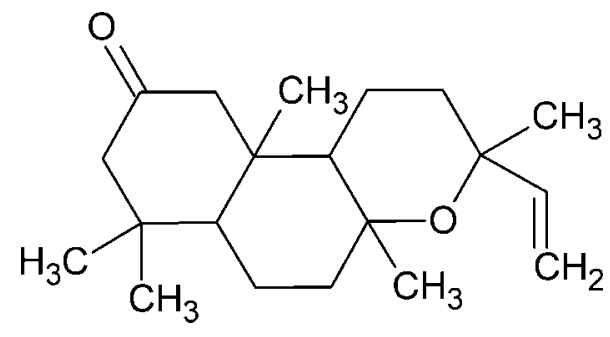




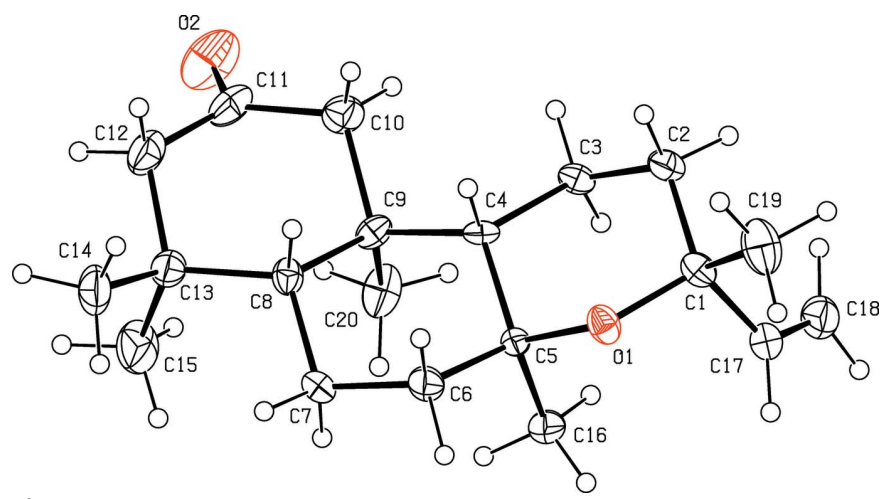

Figure 1

The molecular structure of the title compound, showing the atom labelling and displacement ellipsoids drawn at the $30 \%$ probability level.

\section{Structural commentary}

As shown in Fig. 1, the junction between the two cyclohexane rings $A(\mathrm{C} 8-\mathrm{C} 13)$ and $B(\mathrm{C} 4-\mathrm{C} 9)$ is trans, and the junction for the tetrahydropyran ring $\mathrm{C}(\mathrm{O} 1 / \mathrm{C} 1-\mathrm{C} 5)$ is also trans. The sixmembered carbon rings $A$ and $B$ possess chair conformations [puckering parameters: $Q_{\mathrm{T}}=0.528(7) \AA, \theta=172.6(8)^{\circ}, \varphi=$ $255(6)^{\circ}$ for ring $A$ and $Q_{\mathrm{T}}=0.578(6) \AA, \theta=2.1(6)^{\circ}, \varphi=$ $261(16)^{\circ}$ for ring $\left.B\right]$. The tetrahydropyran ring has a slightly twisted boat conformation [puckering parameters: $Q(2)=$ $0.411(6) \AA$ and $\left.\varphi(2)=81.4(8)^{\circ}\right]$.

\section{Supramolecular features}

In the crystal, molecules pack in helical supramolecular $C(11)$ chains along the $2_{1}$ screw axis running parallel to the $a$ axis, bound by $\mathrm{C}-\mathrm{H} \cdots \mathrm{O}$ hydrogen bonds (Fig. 2 and Table 1 ). The chains are efficiently interlocked in the other two unit-cell directions via van der Waals interactions. Between the chains there are narrow channels which also run along the [100] direction.

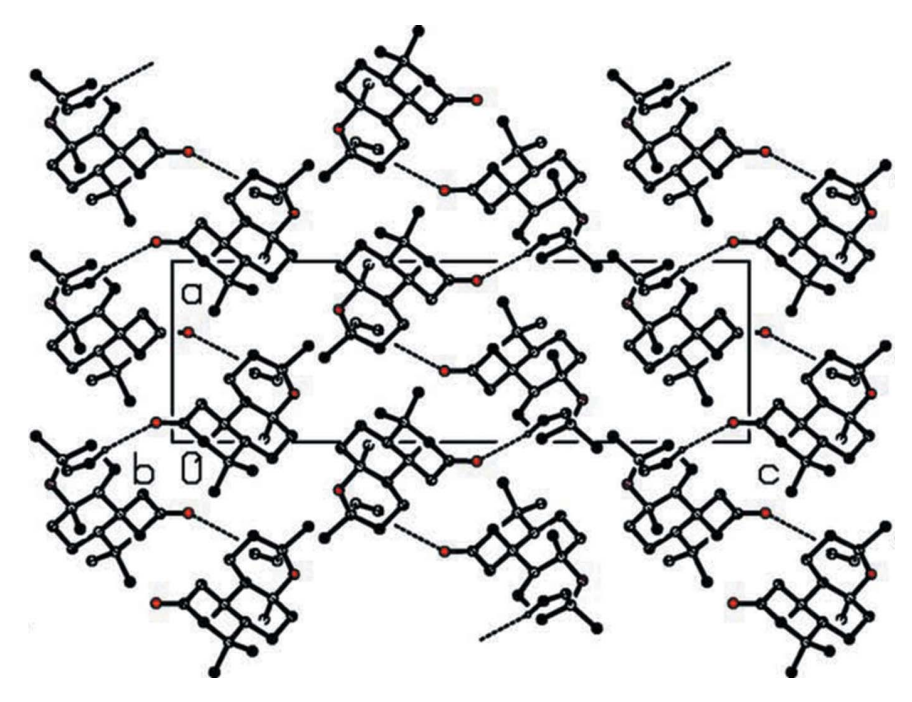

Figure 2

A view along the $a$ axis of the crystal packing of the title compound. $\mathrm{H}$ atoms not involved in these interactions have been omitted for clarity.
Table 1

Hydrogen-bond geometry $\left(\AA,^{\circ}\right)$.

\begin{tabular}{lllll}
\hline$D-\mathrm{H} \cdots A$ & $D-\mathrm{H}$ & $\mathrm{H} \cdots A$ & $D \cdots A$ & $D-\mathrm{H} \cdots A$ \\
\hline $\mathrm{C} 18-\mathrm{H} 18 A \cdots \mathrm{O} 2^{\mathrm{i}}$ & 0.93 & 2.59 & $3.501(9)$ & 167 \\
\hline
\end{tabular}

Symmetry code: (i) $x-\frac{1}{2},-y+\frac{3}{2},-z+1$.

\section{Database survey}

A search of the Cambridge Structural Database (CSD, V5.39, last update February 2018; Groom et al., 2016), for 3-ethenyl3-methyldodecahydro- $1 H$-naphtho[2,1-b]pyran structures, gave 28 hits, all of which present the same basic structural motif as described herein for the title compound. The closest related compound is 2 - $\beta$-hydroxymanoyl oxide [systematic name: $\quad 3,4 a, 7,7,10 \mathrm{a}-$ pentamethyl-3-vinyldodecahydro- $1 \mathrm{H}$ benzo[f]chromen- 9-ol] also isolated from Sideritis perfoliata (UVEVOI; Çelik et al., 2016). Other compounds include, Forskolin $\mathrm{G}$ (systematic name: $1 \alpha$-hydroxy- $6 \beta, 7 \beta$-diacetoxy8,13-epoxylabd-14-en-11-one; CSD refcode ADATUV; Shan et al., 2006), $1 \alpha, 5 \beta$-dihydroxymanoyl oxide, a novel diterpene from Satureja gilliesii (RASXUE; Manríquez et al., 1997), 4ahydroxy-18-normanoyl oxide (GAPZUT; Ybarra et al., 2005), jhanol (GAQBAC; Ybarra et al., 2005), 1R,11S-dihydroxy$8 R, 13 R$-epoxylabd-14-ene (LUDTOU; Stavri et al., 2009) and (-)-paniculatol (NEJHAL; Briand et al., 1997).

In the title compound $\left(P 2_{1} 2_{1} 2_{1}, Z=4\right)$, the molecules pack in helical supramolecular chains along the $2_{1}$ screw axis running parallel to the $a$ axis, bound by one $\mathrm{C}-\mathrm{H} \cdots \mathrm{O}$ hydrogen bond. These chains are efficiently interlocked in the other two unit-cell directions via van der Waals interactions. In the similar compound UVEVOI $\left(P 2_{1} 2_{1} 2_{1}, Z=8\right)$, the asymmetric unit contains two independent molecules. Intermolecular $\mathrm{O}-\mathrm{H} \cdots \mathrm{O}$ hydrogen bonds connect adjacent molecules, forming $C(6)$ helical chains located around a $2_{1}$ screw axis running along the $a$-axis direction. The crystal packing of these chains is governed only by van der Waals interactions. The two asymmetric molecules lead to pseudo- $4_{1}$ symmetry in space group $P 2_{1} 2_{1} 2_{1}$. The crystal structure of the other similar compound UDATUV $\left(P 2_{1}, Z=4\right)$ is stabilized by intermolecular $\mathrm{O}-\mathrm{H} \cdots \mathrm{O}$ and $\mathrm{C}-\mathrm{H} \cdots \mathrm{O}$ hydrogen bonds, which link the molecules into networks approximately parallel to the (110) plane. In the crystal structure of the compound RASXUE $\left(P 2_{1}, Z=4\right)$, no intermolecular hydrogen-bonding interactions were detected, but the $\mathrm{O}-\mathrm{H} \cdots \mathrm{O}$ or $\mathrm{C}-\mathrm{H} \cdots \mathrm{O}$ interactions are possible hydrogen bonds. In GAPZUT $\left(P 2_{1}\right.$, $Z=6$ ), there are three independent molecules in the asymmetric unit. In the crystal, there is no classical hydrogen bonding. The molecular packing is stabilized by van der Waals interactions and no $\pi-\pi$ or $\mathrm{C}-\mathrm{H} \cdots \pi$ interactions are observed. In GAQBAC $\left(P 2_{1}, Z=2\right)$, molecules are connected by $\mathrm{O}-\mathrm{H} \cdots \mathrm{O}$ hydrogen bonds into chains propagating along the $c$-axis direction. Here too, no $\pi-\pi$ or $\mathrm{C}-\mathrm{H} \cdots \pi$ interactions are observed. In LUDTOU $\left(P 2_{1}, Z=4\right)$, the structure contains a water molecule. In the crystal, molecules are connected via $\mathrm{O}-\mathrm{H} \cdots \mathrm{O}$ hydrogen bonds involving the water 


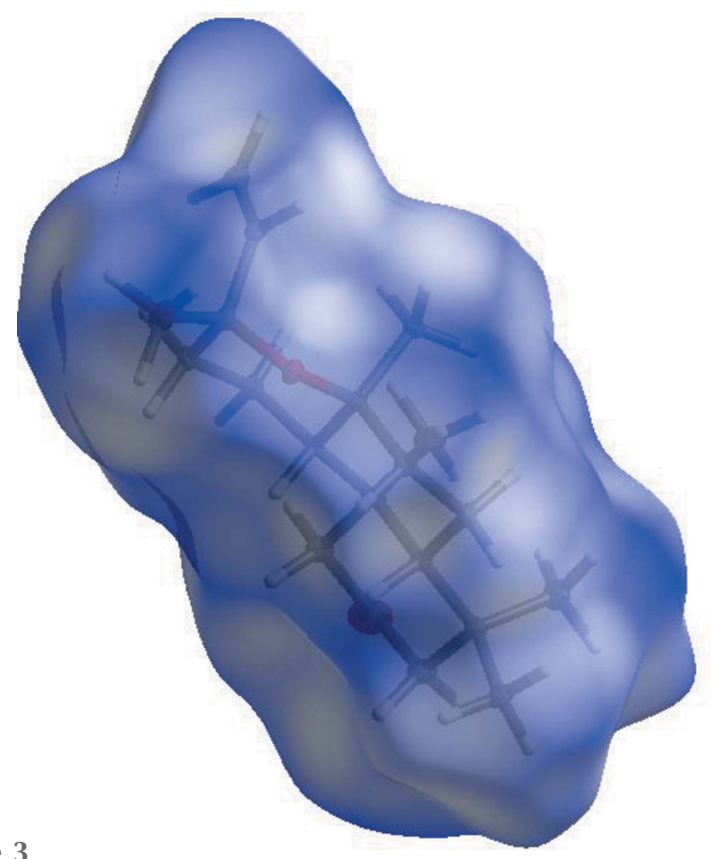

Figure 3

View of the three-dimensional Hirshfeld surface of the title compound mapped with $d_{\text {norm}}$.

molecules, forming a three-dimensional framework. Again no $\pi-\pi$ or $\mathrm{C}-\mathrm{H} \cdots \pi$ interactions are observed.

\section{Hirshfeld surface analysis}

A large range of properties of intermolecular close contacts of a structure can be visualized on the Hirshfeld surface with the program CrystalExplorer (Wolff et al., 2012), including $d_{\mathrm{e}}$ and $d_{\mathrm{i}}$, which represent the distances from a point on the Hirshfeld surface to the nearest atoms outside (external) and inside (internal) the surface, respectively.

Intermolecular distance information on the surface can be condensed into a two-dimensional histogram of $d_{\mathrm{e}}$ and $d_{\mathrm{i}}$, which is a unique identifier for molecules in a crystal structure, and is known as a fingerprint plot (Rohl et al., 2008). Instead of plotting $d_{\mathrm{e}}$ and $d_{\mathrm{i}}$ on the Hirshfeld surface, contact distances are normalized in CrystalExplorer using the van der Waals radius of the appropriate internal $\left(r_{\mathrm{i}}^{\mathrm{vdw}}\right)$ and external $\left(r_{\mathrm{e}}^{\mathrm{vdw}}\right)$ atom of the surface:

$$
d_{\text {norm }}=\left(d_{\mathrm{i}}-r_{\mathrm{i}}^{\mathrm{vdw}}\right) / r_{\mathrm{i}}^{\mathrm{vdw}}+\left(d_{\mathrm{e}}-r_{\mathrm{e}}^{\mathrm{vdw}}\right) / r_{e}^{\mathrm{vdw}} \text {. }
$$

For the title compound, the three-dimensional Hirshfeld surface mapped over $d_{\text {norm }}$ is given in Fig. 3. Contacts with distances equal to the sum of the van der Waals radii are shown in white, and contacts with distances shorter than or longer than the related sum values are shown in red (highlighted contacts) or blue, respectively. Two-dimensional finger print plots showing the occurrence of the various intermolecular contacts are presented in Fig. $4 a-d$. The $\mathrm{H} \cdots \mathrm{H}$ interactions appear in the middle of the scattered points in the two-dimensional fingerprint plots with an overall contribution to the Hirshfeld surface of $86.0 \%$ (Fig. $4 b$ ). The contribution from the $\mathrm{H} \cdots \mathrm{O} / \mathrm{O} \cdots \mathrm{H}$ contacts, corresponding to $\mathrm{C}-\mathrm{H} \cdots \mathrm{O}$ interactions, is represented by a pair of sharp spikes characteristic of a strong hydrogen-bond interaction (12.6\%) (Fig. 4c). The contribution of the other intermolecular contacts to the Hirshfeld surfaces is $\mathrm{H} \cdots \mathrm{C} / \mathrm{C} \cdots \mathrm{H}(1.4 \%)$ (Fig. 4d). The large number of $\mathrm{H} \cdots \mathrm{H}, \mathrm{H} \cdots \mathrm{O} / \mathrm{O} \cdots \mathrm{H}$ and $\mathrm{H} \cdots \mathrm{C} / \mathrm{C} \cdots \mathrm{H}$ interactions suggest that van der Waals inter-
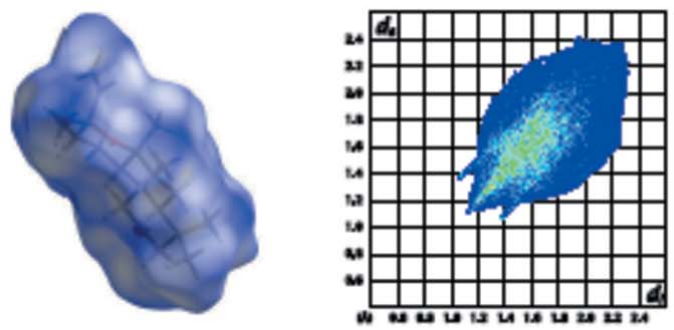

(a) All
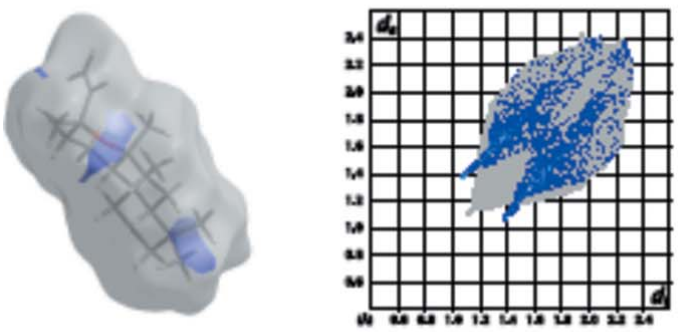

(c) $\mathrm{H} \cdots \mathrm{O} / \mathrm{O} \cdots \mathrm{H}$
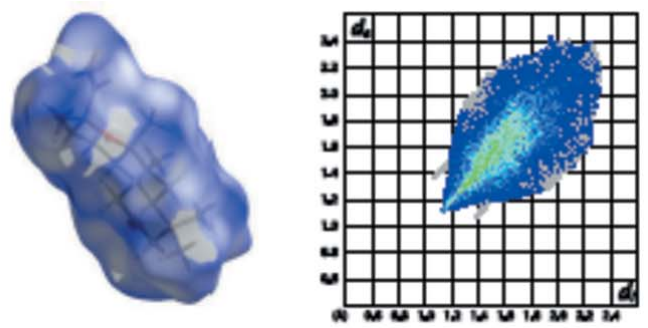

(b) $\mathrm{H} \cdots \mathrm{H}$
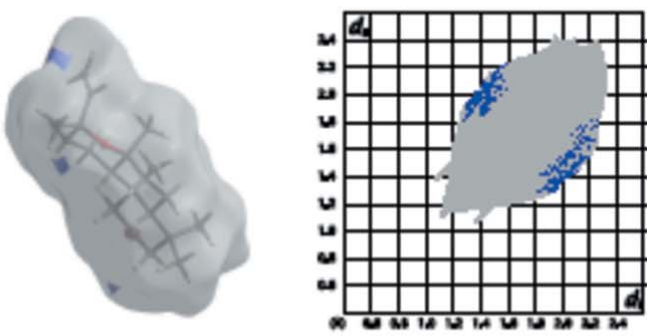

(d) $\mathrm{H} \cdots \mathrm{C} / \mathrm{C} \cdots \mathrm{H}$

Figure 4

The two-dimensional fingerprint plots of the title compound, showing $(a)$ all interactions, and delineated into $(b) \mathrm{H} \cdots \mathrm{H},(c) \mathrm{H} \cdots \mathrm{O}$ and $(d) \mathrm{H} \cdots \mathrm{C}$ interactions $\left[d_{\mathrm{e}}\right.$ and $d_{\mathrm{i}}$ represent the distances from a point on the Hirshfeld surface to the nearest atoms outside (external) and inside (internal) the surface, respectively]. 


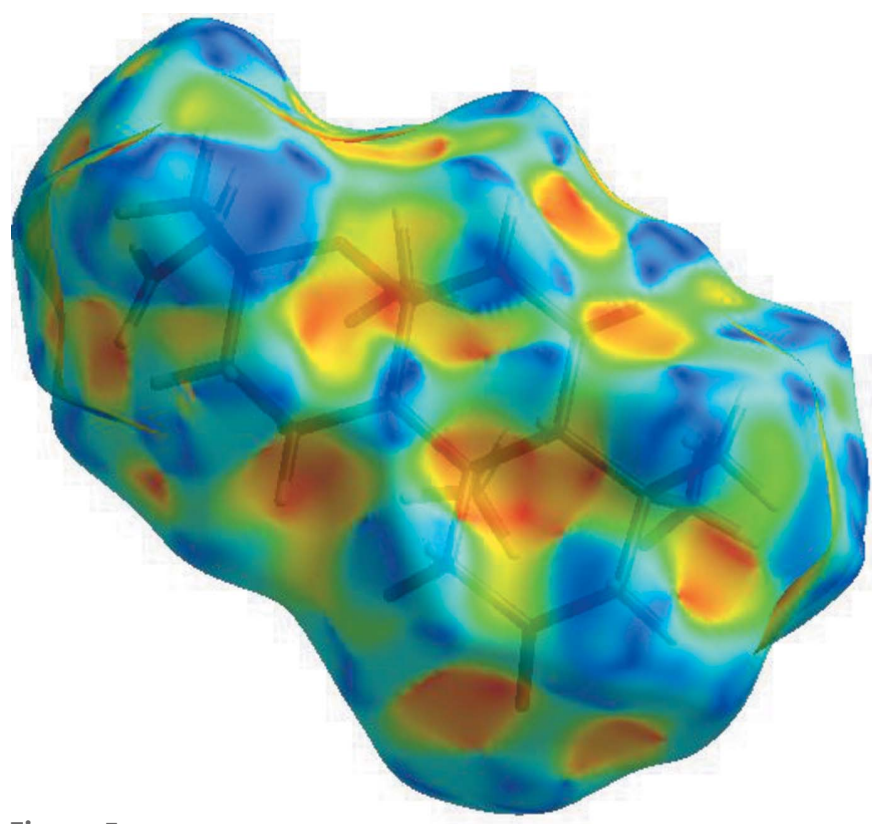

Figure 5

Hirshfeld surface of the title complex plotted over the shape-index.

actions and hydrogen bonding play the major roles in the crystal packing (Hathwar et al., 2015). A view of the Hirshfeld surface of the title complex plotted over the shape-index is given in Fig. 5.

\section{Synthesis and crystallization}

The floral parts of Sideritis perfoliata $(100 \mathrm{~g})$ were extracted with EtOAc $(3 \times 1.0 \mathrm{~L})$. After removal of the solvent in vacuo, the extract $(4.0 \mathrm{~g})$ was subjected to Sephadex LH-20 column chromatography using methanol as the mobile phase at $0.5 \mathrm{ml} /$ min flow rate. According to TLC basis the 6-8th fractions were combined (1.2 g) and separated over silica gel column chromatography using a hexane/EtOAc (6/4) mixture. Fractions 2-4 were combined to give 2-oxo-13-epi-manoyl oxide $(60 \mathrm{mg})$. After removal of the solvent, a white amorphous powder was obtained. The solid was dissolved in acetone and left to stand at room temperature for $12 \mathrm{~h}$. On slow evaporation of the solvent, colourless block-like crystals were obtained.

\section{Refinement details}

Crystal data, data collection and structure refinement details are summarized in Table 2. All $\mathrm{H}$ atoms were placed in geometrically idealized positions and constrained to ride on their parent atoms: $\mathrm{C}-\mathrm{H}=0.93-0.97 \AA$ with $U_{\text {iso }}(\mathrm{H})=$ $1.5 U_{\text {eq }}(\mathrm{C}$-methyl $)$ and $1.2 U_{\text {eq }}(\mathrm{C})$ for other $\mathrm{H}$ atoms. As the title compound is a weak anomalous scatterer, the value of the Flack parameter of -1.1 (10) is meaningless.

\section{Funding information}

This work was supported by the Research Fund of the Scientific Research Project Fund of Cumhuriyet University
Table 2

Experimental details.

\begin{tabular}{|c|c|}
\hline \multicolumn{2}{|l|}{ Crystal data } \\
\hline Chemical formula & $\mathrm{C}_{20} \mathrm{H}_{32} \mathrm{O}_{2}$ \\
\hline$M_{\mathrm{r}}$ & 304.46 \\
\hline Crystal system, space group & Orthorhombic, $P 2_{1} 2_{1} 2_{1}$ \\
\hline Temperature $(\mathrm{K})$ & 296 \\
\hline$a, b, c(\AA)$ & $7.803(2), 9.242(3), 24.952(7)$ \\
\hline$V\left(\AA^{3}\right)$ & $1799.4(9)$ \\
\hline$Z$ & 4 \\
\hline Radiation type & Mo $K \alpha$ \\
\hline$\mu\left(\mathrm{mm}^{-1}\right)$ & 0.07 \\
\hline Crystal size $(\mathrm{mm})$ & $0.12 \times 0.11 \times 0.09$ \\
\hline \multicolumn{2}{|l|}{ Data collection } \\
\hline Diffractometer & Bruker APEXII CCD \\
\hline Absorption correction & $\begin{array}{l}\text { Multi-scan (SADABS; Bruker, } \\
\text { 2007) }\end{array}$ \\
\hline$T_{\min }, T_{\max }$ & $0.596,0.745$ \\
\hline $\begin{array}{l}\text { No. of measured, independent and } \\
\text { observed }[I>2 \sigma(I)] \text { reflections }\end{array}$ & $11666,3530,2120$ \\
\hline$R_{\text {int }}$ & 0.097 \\
\hline$(\sin \theta / \lambda)_{\max }\left(\AA^{-1}\right)$ & 0.626 \\
\hline \multicolumn{2}{|l|}{ Refinement } \\
\hline$R\left[F^{2}>2 \sigma\left(F^{2}\right)\right], w R\left(F^{2}\right), S$ & $0.096,0.186,1.27$ \\
\hline No. of reflections & 3530 \\
\hline No. of parameters & 204 \\
\hline $\mathrm{H}$-atom treatment & $\mathrm{H}$-atom parameters constrained \\
\hline$\Delta \rho_{\max }, \Delta \rho_{\min }\left(\mathrm{e} \AA^{-3}\right)$ & $0.21,-0.25$ \\
\hline
\end{tabular}

Computer programs: APEX2 and SAINT (Bruker, 2007), SHELXS97 (Sheldrick, 2008), SHELXL2014 (Sheldrick, 2015), ORTEP-3 for Windows (Farrugia, 2012) and PLATON (Spek, 2009).

[Project No. F-567 (CUBAP, Sivas, Turkey)]. The authors gratefully acknowledge the financial support received from the Scientific Research Project Fund of Cumhuriyet University.

\section{References}

Briand, A., Kornprobst, J.-M., Al-Easa, H. S., Rizk, A. F. M. \& Toupet, L. (1997). Tetrahedron Lett. 38, 3399-3400.

Bruker (2007). APEX2, SAINT and SADABS. Bruker AXS Inc., Madison, Wisconsin, USA.

Çelik, Í., Ersanlı, C. C., Köseoğlu, R., Akşit, H., Erenler, R., Demirtaş, I. \& Akkurt, M. (2016). Acta Cryst. E72, 1380-1382.

Danesi, F., Saha, S., Kroon, P. A., Glibetić, M., Konić-Ristić, A., D'Antuono, L. F. \& Bordoni, A. (2013). J. Sci. Food Agric. 93, 3558-3564.

Davis, P. H. (1982). Flora of Turkey and the East Aegean Islands. Edinburgh: Edinburgh Univ. Press.

Demirtas, I., Ayhan, B., Sahin, A., Aksit, H., Elmastas, M. \& Telci, I. (2011). Nat. Prod. Res. 25, 1512-1523.

Demirtas, I., Sahin, A., Ayhan, B., Tekin, S. \& Telci, I. (2009). Records of Natural Products, 3, 104-109.

Farrugia, L. J. (2012). J. Appl. Cryst. 45, 849-854.

González-Burgos, E., Carretero, M. \& Gómez-Serranillos, M. (2011). J. Ethnopharmacol. 135, 209-225.

Groom, C. R., Bruno, I. J., Lightfoot, M. P. \& Ward, S. C. (2016). Acta Cryst. B72, 171-179.

Hathwar, V. R., Sist, M., Jørgensen, M. R. V., Mamakhel, A. H., Wang, X., Hoffmann, C. M., Sugimoto, K., Overgaard, J. \& Iversen, B. B. (2015). IUCrJ, 2, 563-574.

Manríquez, V., Labbé, C., Castillo, M. \& Wittke, O. (1997). Acta Cryst. C53, 624-626.

Rohl, A. L., Moret, M., Kaminsky, W., Claborn, K., McKinnon, J. J. \& Kahr, B. (2008). Cryst. Growth Des. 8, 4517-4525. 
Shan, Y.-P., Wang, X.-B. \& Kong, L.-Y. (2006). Acta Cryst. E62, o2408-o2410.

Sheldrick, G. M. (2008). Acta Cryst. A64, 112-122.

Sheldrick, G. M. (2015). Acta Cryst. C71, 3-8.

Spek, A. L. (2009). Acta Cryst. D65, 148-155.

Stavri, M., Paton, A., Skelton, B. W. \& Gibbons, S. (2009). J. Nat. Prod. 72, 1191-1194.
Wolff, S. K., Grimwood, D. J., McKinnon, J. J., Turner, M. J., Jayatilaka, D. \& Spackman, M. A. (2012). Crystal Explorer. University of Western Australia.

Ybarra, M. I., Popich, S., Borkosky, S. A., Asakawa, Y. \& Bardón, A. (2005). J. Nat. Prod. 68, 554-558.

Yiğit Hanoğlu, D., Hanoğlu, A., Güvenir, M., Süer, K., Demirci, B., Başer, K. H. C. \& Yavuz, D. Ö. (2017). J. Essent. Oil Res. 29, 228232. 


\section{supporting information}

Acta Cryst. (2018). E74, 713-717 [https://doi.org/10.1107/S2056989018005807]

\section{Crystal structure and Hirshfeld surface analysis of 2-oxo-13-epi-manoyl oxide isolated from Sideritis perfoliata}

Ísmail Çelik, Zeliha Atioğlu, Huseyin Aksit, Ibrahim Demirtas, Ramazan Erenler and Mehmet Akkurt

Computing details

Data collection: APEX2 (Bruker, 2007); cell refinement: SAINT (Bruker, 2007); data reduction: SAINT (Bruker, 2007); program(s) used to solve structure: SHELXS97 (Sheldrick, 2008); program(s) used to refine structure: SHELXL2014 (Sheldrick, 2015); molecular graphics: ORTEP-3 for Windows (Farrugia, 2012); software used to prepare material for publication: SHELXL2014 (Sheldrick, 2015) and PLATON (Spek, 2009).

3-Ethenyl-3,4a,7,7,10a-pentamethyldodecahydro-9H-benzo[f]chromen-9-one

Crystal data

$\mathrm{C}_{20} \mathrm{H}_{32} \mathrm{O}_{2}$

$M_{r}=304.46$

$F(000)=672$

Orthorhombic, $P 2_{1} 2_{1} 2_{1}$

$D_{\mathrm{x}}=1.124 \mathrm{Mg} \mathrm{m}^{-3}$

Hall symbol: P 2ac 2ab

Mo $K \alpha$ radiation, $\lambda=0.71073 \AA$

$a=7.803$ (2) $\AA$

Cell parameters from 6426 reflections

$b=9.242(3) \AA$

$\theta=3.1-26.4^{\circ}$

$c=24.952(7) \AA$

$\mu=0.07 \mathrm{~mm}^{-1}$

$T=296 \mathrm{~K}$

$V=1799.4(9) \AA^{3}$

Block, colourless

$Z=4$

$0.12 \times 0.11 \times 0.09 \mathrm{~mm}$

\section{Data collection}

Bruker APEXII CCD

diffractometer

$\varphi$ and $\omega$ scans

Absorption correction: multi-scan

(SADABS; Bruker, 2007)

$T_{\min }=0.596, T_{\max }=0.745$

11666 measured reflections

3530 independent reflections

2120 reflections with $I>2 \sigma(I)$

$R_{\text {int }}=0.097$

$\theta_{\text {max }}=26.4^{\circ}, \theta_{\text {min }}=3.1^{\circ}$

$h=-8 \rightarrow 9$

$k=-11 \rightarrow 11$

$l=-30 \rightarrow 31$

Refinement

Refinement on $F^{2}$

Least-squares matrix: full

$R\left[F^{2}>2 \sigma\left(F^{2}\right)\right]=0.096$

$w R\left(F^{2}\right)=0.186$

$S=1.27$

3530 reflections

204 parameters

0 restraints

Primary atom site location: structure-invariant direct methods

Secondary atom site location: difference Fourier map

Hydrogen site location: inferred from neighbouring sites

$\mathrm{H}$-atom parameters constrained 
$w=1 /\left[\sigma^{2}\left(F_{\mathrm{o}}^{2}\right)+(0.0079 P)^{2}+2.0609 P\right]$

where $P=\left(F_{\mathrm{o}}^{2}+2 F_{\mathrm{c}}{ }^{2}\right) / 3$

$(\Delta / \sigma)_{\max }<0.001$

$$
\Delta \rho_{\max }=0.21 \mathrm{e} \AA^{-3}
$$

\section{Special details}

Geometry. Bond distances, angles etc. have been calculated using the rounded fractional coordinates. All su's are estimated from the variances of the (full) variance-covariance matrix. The cell esds are taken into account in the estimation of distances, angles and torsion angles

Refinement. Refinement on $\mathrm{F}^{2}$ for ALL reflections except those flagged by the user for potential systematic errors. Weighted R-factors $\mathrm{WR}$ and all goodnesses of fit $\mathrm{S}$ are based on $\mathrm{F}^{2}$, conventional R-factors $\mathrm{R}$ are based on $\mathrm{F}$, with $\mathrm{F}$ set to zero for negative $\mathrm{F}^{2}$. The observed criterion of $\mathrm{F}^{2}>2 \operatorname{sigma}\left(\mathrm{F}^{2}\right)$ is used only for calculating -R-factor-obs etc. and is not relevant to the choice of reflections for refinement. R-factors based on $\mathrm{F}^{2}$ are statistically about twice as large as those based on F, and R-factors based on ALL data will be even larger.

Fractional atomic coordinates and isotropic or equivalent isotropic displacement parameters $\left(\AA^{2}\right)$

\begin{tabular}{|c|c|c|c|c|}
\hline & $x$ & $y$ & $z$ & $U_{\text {iso }} * / U_{\text {eq }}$ \\
\hline $\mathrm{O} 1$ & $0.2335(5)$ & $0.8443(5)$ & $0.71085(15)$ & $0.0343(16)$ \\
\hline $\mathrm{O} 2$ & $0.3930(9)$ & $0.4214(6)$ & 0.47206 (19) & $0.083(3)$ \\
\hline $\mathrm{C} 1$ & $0.0977(8)$ & $0.9360(7)$ & $0.6904(2)$ & $0.034(2)$ \\
\hline $\mathrm{C} 2$ & $0.0185(8)$ & $0.8669(7)$ & $0.6406(2)$ & $0.037(2)$ \\
\hline $\mathrm{C} 3$ & $0.1511(8)$ & $0.8173(7)$ & $0.5996(2)$ & $0.034(2)$ \\
\hline $\mathrm{C} 4$ & $0.2784(8)$ & $0.7152(6)$ & $0.6273(2)$ & 0.0247 (19) \\
\hline $\mathrm{C} 5$ & $0.3660(8)$ & $0.7939(6)$ & $0.6745(2)$ & 0.0257 (19) \\
\hline C6 & $0.4657(8)$ & $0.6837(7)$ & $0.7072(2)$ & $0.036(2)$ \\
\hline $\mathrm{C} 7$ & $0.5901(8)$ & $0.5959(7)$ & $0.6731(2)$ & $0.035(2)$ \\
\hline $\mathrm{C} 8$ & $0.4971(9)$ & $0.5188(7)$ & $0.6269(2)$ & 0.0310 (19) \\
\hline C9 & $0.4001(9)$ & $0.6277(6)$ & $0.5902(2)$ & 0.0303 (19) \\
\hline $\mathrm{C} 10$ & $0.2898(10)$ & $0.5375(8)$ & $0.5505(2)$ & $0.044(3)$ \\
\hline C11 & $0.3961(11)$ & $0.4285(8)$ & 0.5206 & $0.050(3)$ \\
\hline $\mathrm{C} 12$ & $0.4981(10)$ & $0.3273(7)$ & $0.5547(3)$ & $0.051(3)$ \\
\hline C13 & $0.6085(9)$ & $0.4010(7)$ & $0.5985(3)$ & $0.038(2)$ \\
\hline C14 & $0.6582(10)$ & $0.2831(8)$ & 0.6390 & $0.056(3)$ \\
\hline $\mathrm{C} 15$ & $0.7747(11)$ & $0.4588(9)$ & 0.5732 & $0.067(3)$ \\
\hline $\mathrm{C} 16$ & $0.4820(9)$ & $0.9208(7)$ & 0.6596 & $0.040(2)$ \\
\hline $\mathrm{C} 17$ & $0.1589(9)$ & $1.0879(7)$ & 0.6821 & 0.039 (2) \\
\hline C18 & $0.1195(10)$ & $1.1772(8)$ & $0.6434(3)$ & $0.050(3)$ \\
\hline C19 & $-0.0318(10)$ & $0.9399(8)$ & 0.7360 & $0.059(3)$ \\
\hline $\mathrm{C} 20$ & $0.5154(11)$ & $0.7268(7)$ & $0.5559(3)$ & $0.049(3)$ \\
\hline $\mathrm{H} 2 \mathrm{~A}$ & -0.04990 & 0.78430 & 0.65140 & $0.0440^{*}$ \\
\hline $\mathrm{H} 2 \mathrm{~B}$ & -0.05760 & 0.93630 & 0.62370 & $0.0440 *$ \\
\hline $\mathrm{H} 3 \mathrm{~A}$ & 0.21130 & 0.90030 & 0.58510 & $0.0410 *$ \\
\hline H3B & 0.09470 & 0.76740 & 0.57030 & $0.0410 *$ \\
\hline H4 & 0.20610 & 0.64180 & 0.64440 & $0.0300 *$ \\
\hline H6A & 0.38570 & 0.61840 & 0.72450 & $0.0430 *$ \\
\hline H6B & 0.52920 & 0.73360 & 0.73500 & $0.0430 *$ \\
\hline $\mathrm{H} 7 \mathrm{~A}$ & 0.64680 & 0.52460 & 0.69540 & $0.0420^{*}$ \\
\hline H7B & 0.67720 & 0.65970 & 0.65850 & $0.0420 *$ \\
\hline H8 & 0.40580 & 0.46380 & 0.64450 & $0.0370^{*}$ \\
\hline
\end{tabular}




$\begin{array}{lllll}\text { H10A } & 0.20050 & 0.48750 & 0.57030 & 0.0530^{*} \\ \text { H10B } & 0.23500 & 0.60210 & 0.52520 & 0.0530^{*} \\ \text { H12A } & 0.57310 & 0.27130 & 0.53160 & 0.0610^{*} \\ \text { H12B } & 0.41990 & 0.26020 & 0.57190 & 0.0610^{*} \\ \text { H14A } & 0.55750 & 0.25030 & 0.65750 & 0.0840^{*} \\ \text { H14B } & 0.73820 & 0.32210 & 0.66440 & 0.0840^{*} \\ \text { H14C } & 0.70990 & 0.20330 & 0.62040 & 0.0840^{*} \\ \text { H15A } & 0.83750 & 0.51340 & 0.59950 & 0.1000^{*} \\ \text { H15B } & 0.74710 & 0.52010 & 0.54340 & 0.1000^{*} \\ \text { H15C } & 0.84340 & 0.37910 & 0.56110 & 0.1000^{*} \\ \text { H16A } & 0.42730 & 0.97830 & 0.63240 & 0.0600^{*} \\ \text { H16B } & 0.58910 & 0.88470 & 0.64610 & 0.0600^{*} \\ \text { H16C } & 0.50240 & 0.97930 & 0.69070 & 0.0600^{*} \\ \text { H17 } & 0.23470 & 1.12310 & 0.70770 & 0.0470^{*} \\ \text { H18A } & 0.04430 & 1.14840 & 0.61660 & 0.0600^{*} \\ \text { H18B } & 0.16670 & 1.26960 & 0.64280 & 0.0600^{*} \\ \text { H19A } & -0.07240 & 0.84370 & 0.74300 & 0.0890^{*} \\ \text { H19B } & -0.12660 & 1.00050 & 0.72610 & 0.0890^{*} \\ \text { H19C } & 0.02180 & 0.97810 & 0.76760 & 0.0890^{*} \\ \text { H20A } & 0.44920 & 0.80690 & 0.54280 & 0.0740^{*} \\ \text { H20B } & 0.56040 & 0.67290 & 0.52620 & 0.0740^{*} \\ \text { H20C } & 0.60830 & 0.76240 & 0.57740 & 0.0740^{*}\end{array}$

Atomic displacement parameters $\left(\AA^{2}\right)$

\begin{tabular}{lllllll}
\hline & $U^{11}$ & $U^{22}$ & $U^{\beta 3}$ & $U^{12}$ & $U^{13}$ & $U^{23}$ \\
\hline $\mathrm{O} 1$ & $0.032(3)$ & $0.044(3)$ & $0.027(2)$ & $0.010(2)$ & $0.0025(19)$ & $0.000(2)$ \\
$\mathrm{O} 2$ & $0.132(6)$ & $0.082(4)$ & $0.035(3)$ & $-0.004(4)$ & $0.005(3)$ & $-0.019(3)$ \\
$\mathrm{C} 1$ & $0.025(4)$ & $0.040(4)$ & $0.038(3)$ & $0.001(3)$ & $0.002(3)$ & $0.001(3)$ \\
$\mathrm{C} 2$ & $0.025(4)$ & $0.039(4)$ & $0.047(4)$ & $-0.002(3)$ & $-0.005(3)$ & $0.000(3)$ \\
$\mathrm{C} 3$ & $0.032(4)$ & $0.040(4)$ & $0.030(3)$ & $0.003(3)$ & $-0.014(3)$ & $0.000(3)$ \\
$\mathrm{C} 4$ & $0.022(3)$ & $0.031(4)$ & $0.021(3)$ & $-0.010(3)$ & $-0.002(3)$ & $0.000(3)$ \\
$\mathrm{C} 5$ & $0.023(3)$ & $0.027(4)$ & $0.027(3)$ & $0.002(3)$ & $-0.003(3)$ & $-0.006(3)$ \\
$\mathrm{C} 6$ & $0.039(4)$ & $0.041(4)$ & $0.028(3)$ & $0.007(4)$ & $-0.011(3)$ & $-0.003(3)$ \\
C7 & $0.025(4)$ & $0.038(4)$ & $0.043(4)$ & $0.004(3)$ & $-0.007(3)$ & $-0.003(3)$ \\
C8 & $0.030(4)$ & $0.030(3)$ & $0.033(3)$ & $0.003(3)$ & $0.008(3)$ & $0.000(3)$ \\
C9 & $0.041(4)$ & $0.028(3)$ & $0.022(3)$ & $-0.005(3)$ & $0.001(3)$ & $-0.002(3)$ \\
C10 & $0.051(5)$ & $0.046(5)$ & $0.035(4)$ & $0.000(4)$ & $-0.010(3)$ & $-0.002(3)$ \\
C11 & $0.066(5)$ & $0.042(4)$ & $0.042(4)$ & $-0.010(4)$ & $0.004(4)$ & $-0.017(4)$ \\
C12 & $0.058(5)$ & $0.040(4)$ & $0.054(4)$ & $0.000(4)$ & $0.010(4)$ & $-0.016(4)$ \\
C13 & $0.033(4)$ & $0.033(4)$ & $0.049(4)$ & $-0.003(3)$ & $0.007(3)$ & $-0.004(3)$ \\
C14 & $0.056(5)$ & $0.037(4)$ & $0.076(6)$ & $0.016(4)$ & $0.004(4)$ & $-0.002(4)$ \\
C15 & $0.052(5)$ & $0.063(6)$ & $0.086(6)$ & $0.004(5)$ & $0.029(5)$ & $-0.011(5)$ \\
C16 & $0.031(4)$ & $0.035(4)$ & $0.055(4)$ & $-0.005(4)$ & $0.000(3)$ & $-0.007(3)$ \\
C17 & $0.034(4)$ & $0.035(4)$ & $0.048(4)$ & $0.006(3)$ & $-0.003(3)$ & $-0.005(4)$ \\
C18 & $0.043(5)$ & $0.039(4)$ & $0.068(5)$ & $0.001(4)$ & $0.004(4)$ & $0.000(4)$ \\
C19 & $0.053(5)$ & $0.068(5)$ & $0.057(5)$ & $0.017(5)$ & $0.024(4)$ & $0.005(4)$ \\
C20 & $0.070(6)$ & $0.040(4)$ & $0.038(4)$ & $0.004(4)$ & $0.024(4)$ & $0.004(3)$ \\
& & & & & &
\end{tabular}


Geometric parameters $\left(\AA,{ }^{\circ}\right)$

\begin{tabular}{|c|c|c|c|}
\hline $\mathrm{O} 1-\mathrm{C} 1$ & $1.450(7)$ & $\mathrm{C} 4-\mathrm{H} 4$ & 0.9800 \\
\hline $\mathrm{O} 1-\mathrm{C} 5$ & $1.452(7)$ & C6-H6A & 0.9700 \\
\hline $\mathrm{O} 2-\mathrm{C} 11$ & $1.213(9)$ & C6-H6B & 0.9700 \\
\hline $\mathrm{C} 1-\mathrm{C} 2$ & $1.528(8)$ & C7-H7A & 0.9700 \\
\hline $\mathrm{C} 1-\mathrm{C} 17$ & $1.497(9)$ & C7-H7B & 0.9700 \\
\hline $\mathrm{C} 1-\mathrm{C} 19$ & $1.522(9)$ & $\mathrm{C} 8-\mathrm{H} 8$ & 0.9800 \\
\hline $\mathrm{C} 2-\mathrm{C} 3$ & $1.526(8)$ & $\mathrm{C} 10-\mathrm{H} 10 \mathrm{~A}$ & 0.9700 \\
\hline $\mathrm{C} 3-\mathrm{C} 4$ & $1.535(8)$ & $\mathrm{C} 10-\mathrm{H} 10 \mathrm{~B}$ & 0.9700 \\
\hline $\mathrm{C} 4-\mathrm{C} 5$ & $1.544(8)$ & $\mathrm{C} 12-\mathrm{H} 12 \mathrm{~A}$ & 0.9700 \\
\hline $\mathrm{C} 4-\mathrm{C} 9$ & $1.553(8)$ & $\mathrm{C} 12-\mathrm{H} 12 \mathrm{~B}$ & 0.9700 \\
\hline $\mathrm{C} 5-\mathrm{C} 6$ & $1.519(8)$ & $\mathrm{C} 14-\mathrm{H} 14 \mathrm{~A}$ & 0.9600 \\
\hline $\mathrm{C} 5-\mathrm{C} 16$ & $1.527(9)$ & $\mathrm{C} 14-\mathrm{H} 14 \mathrm{~B}$ & 0.9600 \\
\hline $\mathrm{C} 6-\mathrm{C} 7$ & $1.525(8)$ & $\mathrm{C} 14-\mathrm{H} 14 \mathrm{C}$ & 0.9600 \\
\hline $\mathrm{C} 7-\mathrm{C} 8$ & $1.537(8)$ & $\mathrm{C} 15-\mathrm{H} 15 \mathrm{~A}$ & 0.9600 \\
\hline $\mathrm{C} 8-\mathrm{C} 9$ & $1.557(8)$ & C15-H15B & 0.9600 \\
\hline $\mathrm{C} 8-\mathrm{C} 13$ & $1.563(9)$ & $\mathrm{C} 15-\mathrm{H} 15 \mathrm{C}$ & 0.9600 \\
\hline $\mathrm{C} 9-\mathrm{C} 10$ & $1.555(9)$ & C16-H16A & 0.9600 \\
\hline $\mathrm{C} 9-\mathrm{C} 20$ & $1.543(10)$ & C16-H16B & 0.9600 \\
\hline $\mathrm{C} 10-\mathrm{C} 11$ & $1.503(10)$ & $\mathrm{C} 16-\mathrm{H} 16 \mathrm{C}$ & 0.9600 \\
\hline $\mathrm{C} 11-\mathrm{C} 12$ & $1.494(11)$ & C17-H17 & 0.9300 \\
\hline $\mathrm{C} 12-\mathrm{C} 13$ & $1.549(10)$ & $\mathrm{C} 18-\mathrm{H} 18 \mathrm{~A}$ & 0.9300 \\
\hline $\mathrm{C} 13-\mathrm{C} 14$ & $1.536(10)$ & C18-H18B & 0.9300 \\
\hline $\mathrm{C} 13-\mathrm{C} 15$ & $1.538(11)$ & C19-H19A & 0.9600 \\
\hline $\mathrm{C} 17-\mathrm{C} 18$ & $1.307(10)$ & C19-H19B & 0.9600 \\
\hline $\mathrm{C} 2-\mathrm{H} 2 \mathrm{~A}$ & 0.9700 & C19-H19C & 0.9600 \\
\hline $\mathrm{C} 2-\mathrm{H} 2 \mathrm{~B}$ & 0.9700 & $\mathrm{C} 20-\mathrm{H} 20 \mathrm{~A}$ & 0.9600 \\
\hline $\mathrm{C} 3-\mathrm{H} 3 \mathrm{~A}$ & 0.9700 & $\mathrm{C} 20-\mathrm{H} 20 \mathrm{~B}$ & 0.9600 \\
\hline $\mathrm{C} 3-\mathrm{H} 3 \mathrm{~B}$ & 0.9700 & $\mathrm{C} 20-\mathrm{H} 20 \mathrm{C}$ & 0.9600 \\
\hline $\mathrm{C} 1-\mathrm{O} 1-\mathrm{C} 5$ & $119.2(4)$ & $\mathrm{C} 7-\mathrm{C} 6-\mathrm{H} 6 \mathrm{~A}$ & 109.00 \\
\hline $\mathrm{O} 1-\mathrm{C} 1-\mathrm{C} 2$ & $109.7(5)$ & $\mathrm{C} 7-\mathrm{C} 6-\mathrm{H} 6 \mathrm{~B}$ & 109.00 \\
\hline $\mathrm{O} 1-\mathrm{C} 1-\mathrm{C} 17$ & $111.3(5)$ & $\mathrm{H} 6 \mathrm{~A}-\mathrm{C} 6-\mathrm{H} 6 \mathrm{~B}$ & 108.00 \\
\hline $\mathrm{O} 1-\mathrm{C} 1-\mathrm{C} 19$ & $103.7(5)$ & $\mathrm{C} 6-\mathrm{C} 7-\mathrm{H} 7 \mathrm{~A}$ & 109.00 \\
\hline $\mathrm{C} 2-\mathrm{C} 1-\mathrm{C} 17$ & $114.1(5)$ & $\mathrm{C} 6-\mathrm{C} 7-\mathrm{H} 7 \mathrm{~B}$ & 109.00 \\
\hline $\mathrm{C} 2-\mathrm{C} 1-\mathrm{C} 19$ & $110.5(5)$ & $\mathrm{C} 8-\mathrm{C} 7-\mathrm{H} 7 \mathrm{~A}$ & 109.00 \\
\hline $\mathrm{C} 17-\mathrm{C} 1-\mathrm{C} 19$ & $107.0(5)$ & $\mathrm{C} 8-\mathrm{C} 7-\mathrm{H} 7 \mathrm{~B}$ & 109.00 \\
\hline $\mathrm{C} 1-\mathrm{C} 2-\mathrm{C} 3$ & $113.4(5)$ & $\mathrm{H} 7 \mathrm{~A}-\mathrm{C} 7-\mathrm{H} 7 \mathrm{~B}$ & 108.00 \\
\hline $\mathrm{C} 2-\mathrm{C} 3-\mathrm{C} 4$ & $108.8(4)$ & $\mathrm{C} 7-\mathrm{C} 8-\mathrm{H} 8$ & 104.00 \\
\hline $\mathrm{C} 3-\mathrm{C} 4-\mathrm{C} 5$ & $109.9(5)$ & $\mathrm{C} 9-\mathrm{C} 8-\mathrm{H} 8$ & 104.00 \\
\hline $\mathrm{C} 3-\mathrm{C} 4-\mathrm{C} 9$ & $116.6(4)$ & $\mathrm{C} 13-\mathrm{C} 8-\mathrm{H} 8$ & 104.00 \\
\hline $\mathrm{C} 5-\mathrm{C} 4-\mathrm{C} 9$ & $115.4(5)$ & $\mathrm{C} 9-\mathrm{C} 10-\mathrm{H} 10 \mathrm{~A}$ & 109.00 \\
\hline $\mathrm{O} 1-\mathrm{C} 5-\mathrm{C} 4$ & $108.2(5)$ & $\mathrm{C} 9-\mathrm{C} 10-\mathrm{H} 10 \mathrm{~B}$ & 109.00 \\
\hline $\mathrm{O} 1-\mathrm{C} 5-\mathrm{C} 6$ & $104.1(4)$ & $\mathrm{C} 11-\mathrm{C} 10-\mathrm{H} 10 \mathrm{~A}$ & 109.00 \\
\hline $\mathrm{O} 1-\mathrm{C} 5-\mathrm{C} 16$ & $109.1(5)$ & $\mathrm{C} 11-\mathrm{C} 10-\mathrm{H} 10 \mathrm{~B}$ & 109.00 \\
\hline $\mathrm{C} 4-\mathrm{C} 5-\mathrm{C} 6$ & $108.7(5)$ & $\mathrm{H} 10 \mathrm{~A}-\mathrm{C} 10-\mathrm{H} 10 \mathrm{~B}$ & 108.00 \\
\hline $\mathrm{C} 4-\mathrm{C} 5-\mathrm{C} 16$ & $116.0(5)$ & $\mathrm{C} 11-\mathrm{C} 12-\mathrm{H} 12 \mathrm{~A}$ & 108.00 \\
\hline
\end{tabular}




\begin{tabular}{|c|c|c|c|}
\hline $\mathrm{C} 6-\mathrm{C} 5-\mathrm{C} 16$ & $110.0(5)$ & $\mathrm{C} 11-\mathrm{C} 12-\mathrm{H} 12 \mathrm{~B}$ & 108.00 \\
\hline $\mathrm{C} 5-\mathrm{C} 6-\mathrm{C} 7$ & $112.5(4)$ & $\mathrm{C} 13-\mathrm{C} 12-\mathrm{H} 12 \mathrm{~A}$ & 109.00 \\
\hline $\mathrm{C} 6-\mathrm{C} 7-\mathrm{C} 8$ & $111.4(5)$ & $\mathrm{C} 13-\mathrm{C} 12-\mathrm{H} 12 \mathrm{~B}$ & 109.00 \\
\hline $\mathrm{C} 7-\mathrm{C} 8-\mathrm{C} 9$ & $111.8(5)$ & $\mathrm{H} 12 \mathrm{~A}-\mathrm{C} 12-\mathrm{H} 12 \mathrm{~B}$ & 108.00 \\
\hline $\mathrm{C} 7-\mathrm{C} 8-\mathrm{C} 13$ & $113.6(6)$ & $\mathrm{C} 13-\mathrm{C} 14-\mathrm{H} 14 \mathrm{~A}$ & 109.00 \\
\hline $\mathrm{C} 9-\mathrm{C} 8-\mathrm{C} 13$ & $117.0(5)$ & $\mathrm{C} 13-\mathrm{C} 14-\mathrm{H} 14 \mathrm{~B}$ & 109.00 \\
\hline $\mathrm{C} 4-\mathrm{C} 9-\mathrm{C} 8$ & $106.5(4)$ & $\mathrm{C} 13-\mathrm{C} 14-\mathrm{H} 14 \mathrm{C}$ & 109.00 \\
\hline $\mathrm{C} 4-\mathrm{C} 9-\mathrm{C} 10$ & $108.7(5)$ & $\mathrm{H} 14 \mathrm{~A}-\mathrm{C} 14-\mathrm{H} 14 \mathrm{~B}$ & 109.00 \\
\hline $\mathrm{C} 4-\mathrm{C} 9-\mathrm{C} 20$ & $112.2(5)$ & $\mathrm{H} 14 \mathrm{~A}-\mathrm{C} 14-\mathrm{H} 14 \mathrm{C}$ & 109.00 \\
\hline $\mathrm{C} 8-\mathrm{C} 9-\mathrm{C} 10$ & $107.3(5)$ & $\mathrm{H} 14 \mathrm{~B}-\mathrm{C} 14-\mathrm{H} 14 \mathrm{C}$ & 110.00 \\
\hline $\mathrm{C} 8-\mathrm{C} 9-\mathrm{C} 20$ & $115.2(6)$ & $\mathrm{C} 13-\mathrm{C} 15-\mathrm{H} 15 \mathrm{~A}$ & 109.00 \\
\hline $\mathrm{C} 10-\mathrm{C} 9-\mathrm{C} 20$ & $106.7(5)$ & $\mathrm{C} 13-\mathrm{C} 15-\mathrm{H} 15 \mathrm{~B}$ & 110.00 \\
\hline $\mathrm{C} 9-\mathrm{C} 10-\mathrm{C} 11$ & $111.7(6)$ & $\mathrm{C} 13-\mathrm{C} 15-\mathrm{H} 15 \mathrm{C}$ & 109.00 \\
\hline $\mathrm{O} 2-\mathrm{C} 11-\mathrm{C} 10$ & $121.4(7)$ & $\mathrm{H} 15 \mathrm{~A}-\mathrm{C} 15-\mathrm{H} 15 \mathrm{~B}$ & 109.00 \\
\hline $\mathrm{O} 2-\mathrm{C} 11-\mathrm{C} 12$ & $123.1(7)$ & $\mathrm{H} 15 \mathrm{~A}-\mathrm{C} 15-\mathrm{H} 15 \mathrm{C}$ & 109.00 \\
\hline $\mathrm{C} 10-\mathrm{C} 11-\mathrm{C} 12$ & $115.5(6)$ & $\mathrm{H} 15 \mathrm{~B}-\mathrm{C} 15-\mathrm{H} 15 \mathrm{C}$ & 110.00 \\
\hline $\mathrm{C} 11-\mathrm{C} 12-\mathrm{C} 13$ & $115.0(6)$ & $\mathrm{C} 5-\mathrm{C} 16-\mathrm{H} 16 \mathrm{~A}$ & 109.00 \\
\hline $\mathrm{C} 8-\mathrm{C} 13-\mathrm{C} 12$ & $108.5(6)$ & $\mathrm{C} 5-\mathrm{C} 16-\mathrm{H} 16 \mathrm{~B}$ & 110.00 \\
\hline $\mathrm{C} 8-\mathrm{C} 13-\mathrm{C} 14$ & $109.7(6)$ & $\mathrm{C} 5-\mathrm{C} 16-\mathrm{H} 16 \mathrm{C}$ & 110.00 \\
\hline $\mathrm{C} 8-\mathrm{C} 13-\mathrm{C} 15$ & $114.4(6)$ & $\mathrm{H} 16 \mathrm{~A}-\mathrm{C} 16-\mathrm{H} 16 \mathrm{~B}$ & 109.00 \\
\hline $\mathrm{C} 12-\mathrm{C} 13-\mathrm{C} 14$ & $107.0(5)$ & $\mathrm{H} 16 \mathrm{~A}-\mathrm{C} 16-\mathrm{H} 16 \mathrm{C}$ & 109.00 \\
\hline $\mathrm{C} 12-\mathrm{C} 13-\mathrm{C} 15$ & $109.4(6)$ & $\mathrm{H} 16 \mathrm{~B}-\mathrm{C} 16-\mathrm{H} 16 \mathrm{C}$ & 110.00 \\
\hline $\mathrm{C} 14-\mathrm{C} 13-\mathrm{C} 15$ & $107.7(6)$ & $\mathrm{C} 1-\mathrm{C} 17-\mathrm{H} 17$ & 116.00 \\
\hline $\mathrm{C} 1-\mathrm{C} 17-\mathrm{C} 18$ & $128.3(7)$ & $\mathrm{C} 18-\mathrm{C} 17-\mathrm{H} 17$ & 116.00 \\
\hline $\mathrm{C} 1-\mathrm{C} 2-\mathrm{H} 2 \mathrm{~A}$ & 109.00 & $\mathrm{C} 17-\mathrm{C} 18-\mathrm{H} 18 \mathrm{~A}$ & 120.00 \\
\hline $\mathrm{C} 1-\mathrm{C} 2-\mathrm{H} 2 \mathrm{~B}$ & 109.00 & $\mathrm{C} 17-\mathrm{C} 18-\mathrm{H} 18 \mathrm{~B}$ & 120.00 \\
\hline $\mathrm{C} 3-\mathrm{C} 2-\mathrm{H} 2 \mathrm{~A}$ & 109.00 & $\mathrm{H} 18 \mathrm{~A}-\mathrm{C} 18-\mathrm{H} 18 \mathrm{~B}$ & 120.00 \\
\hline $\mathrm{C} 3-\mathrm{C} 2-\mathrm{H} 2 \mathrm{~B}$ & 109.00 & $\mathrm{C} 1-\mathrm{C} 19-\mathrm{H} 19 \mathrm{~A}$ & 109.00 \\
\hline $\mathrm{H} 2 \mathrm{~A}-\mathrm{C} 2-\mathrm{H} 2 \mathrm{~B}$ & 108.00 & $\mathrm{C} 1-\mathrm{C} 19-\mathrm{H} 19 \mathrm{~B}$ & 109.00 \\
\hline $\mathrm{C} 2-\mathrm{C} 3-\mathrm{H} 3 \mathrm{~A}$ & 110.00 & $\mathrm{C} 1-\mathrm{C} 19-\mathrm{H} 19 \mathrm{C}$ & 109.00 \\
\hline $\mathrm{C} 2-\mathrm{C} 3-\mathrm{H} 3 \mathrm{~B}$ & 110.00 & $\mathrm{H} 19 \mathrm{~A}-\mathrm{C} 19-\mathrm{H} 19 \mathrm{~B}$ & 109.00 \\
\hline $\mathrm{C} 4-\mathrm{C} 3-\mathrm{H} 3 \mathrm{~A}$ & 110.00 & $\mathrm{H} 19 \mathrm{~A}-\mathrm{C} 19-\mathrm{H} 19 \mathrm{C}$ & 110.00 \\
\hline $\mathrm{C} 4-\mathrm{C} 3-\mathrm{H} 3 \mathrm{~B}$ & 110.00 & $\mathrm{H} 19 \mathrm{~B}-\mathrm{C} 19-\mathrm{H} 19 \mathrm{C}$ & 109.00 \\
\hline $\mathrm{H} 3 \mathrm{~A}-\mathrm{C} 3-\mathrm{H} 3 \mathrm{~B}$ & 108.00 & $\mathrm{C} 9-\mathrm{C} 20-\mathrm{H} 20 \mathrm{~A}$ & 109.00 \\
\hline $\mathrm{C} 3-\mathrm{C} 4-\mathrm{H} 4$ & 104.00 & $\mathrm{C} 9-\mathrm{C} 20-\mathrm{H} 20 \mathrm{~B}$ & 109.00 \\
\hline $\mathrm{C} 5-\mathrm{C} 4-\mathrm{H} 4$ & 104.00 & $\mathrm{C} 9-\mathrm{C} 20-\mathrm{H} 20 \mathrm{C}$ & 109.00 \\
\hline $\mathrm{C} 9-\mathrm{C} 4-\mathrm{H} 4$ & 105.00 & $\mathrm{H} 20 \mathrm{~A}-\mathrm{C} 20-\mathrm{H} 20 \mathrm{~B}$ & 110.00 \\
\hline $\mathrm{C} 5-\mathrm{C} 6-\mathrm{H} 6 \mathrm{~A}$ & 109.00 & $\mathrm{H} 20 \mathrm{~A}-\mathrm{C} 20-\mathrm{H} 20 \mathrm{C}$ & 109.00 \\
\hline $\mathrm{C} 5-\mathrm{C} 6-\mathrm{H} 6 \mathrm{~B}$ & 109.00 & $\mathrm{H} 20 \mathrm{~B}-\mathrm{C} 20-\mathrm{H} 20 \mathrm{C}$ & 109.00 \\
\hline $\mathrm{C} 5-\mathrm{O} 1-\mathrm{C} 1-\mathrm{C} 2$ & $50.8(7)$ & $\mathrm{C} 4-\mathrm{C} 5-\mathrm{C} 6-\mathrm{C} 7$ & $53.8(7)$ \\
\hline $\mathrm{C} 5-\mathrm{O} 1-\mathrm{C} 1-\mathrm{C} 17$ & $-76.5(6)$ & $\mathrm{C} 16-\mathrm{C} 5-\mathrm{C} 6-\mathrm{C} 7$ & $-74.3(6)$ \\
\hline $\mathrm{C} 5-\mathrm{O} 1-\mathrm{C} 1-\mathrm{C} 19$ & $168.8(5)$ & $\mathrm{C} 5-\mathrm{C} 6-\mathrm{C} 7-\mathrm{C} 8$ & $-56.6(7)$ \\
\hline $\mathrm{C} 1-\mathrm{O} 1-\mathrm{C} 5-\mathrm{C} 6$ & $-171.1(5)$ & $\mathrm{C} 6-\mathrm{C} 7-\mathrm{C} 8-\mathrm{C} 9$ & $57.8(7)$ \\
\hline $\mathrm{C} 1-\mathrm{O} 1-\mathrm{C} 5-\mathrm{C} 16$ & $71.5(6)$ & $\mathrm{C} 6-\mathrm{C} 7-\mathrm{C} 8-\mathrm{C} 13$ & $-167.1(5)$ \\
\hline $\mathrm{C} 1-\mathrm{O} 1-\mathrm{C} 5-\mathrm{C} 4$ & $-55.5(6)$ & $\mathrm{C} 7-\mathrm{C} 8-\mathrm{C} 9-\mathrm{C} 4$ & $-55.5(6)$ \\
\hline $\mathrm{C} 19-\mathrm{C} 1-\mathrm{C} 2-\mathrm{C} 3$ & $-162.4(5)$ & $\mathrm{C} 7-\mathrm{C} 8-\mathrm{C} 9-\mathrm{C} 10$ & $-171.7(5)$ \\
\hline $\mathrm{C} 2-\mathrm{C} 1-\mathrm{C} 17-\mathrm{C} 18$ & $17.1(10)$ & $\mathrm{C} 7-\mathrm{C} 8-\mathrm{C} 9-\mathrm{C} 20$ & $69.6(6)$ \\
\hline
\end{tabular}




$\begin{array}{llll}\mathrm{C} 19-\mathrm{C} 1-\mathrm{C} 17-\mathrm{C} 18 & -105.4(8) & \mathrm{C} 13-\mathrm{C} 8-\mathrm{C} 9-\mathrm{C} 4 & 171.1(5) \\ \mathrm{O} 1-\mathrm{C} 1-\mathrm{C} 17-\mathrm{C} 18 & 142.0(7) & \mathrm{C} 13-\mathrm{C} 8-\mathrm{C} 9-\mathrm{C} 10 & 54.9(7) \\ \mathrm{O} 1-\mathrm{C} 1-\mathrm{C} 2-\mathrm{C} 3 & -48.8(6) & \mathrm{C} 13-\mathrm{C} 8-\mathrm{C} 9-\mathrm{C} 20 & -63.8(7) \\ \mathrm{C} 17-\mathrm{C} 1-\mathrm{C} 2-\mathrm{C} 3 & 76.9(7) & \mathrm{C} 7-\mathrm{C} 8-\mathrm{C} 13-\mathrm{C} 12 & 177.6(5) \\ \mathrm{C} 1-\mathrm{C} 2-\mathrm{C} 3-\mathrm{C} 4 & 55.0(7) & \mathrm{C} 7-\mathrm{C} 8-\mathrm{C} 13-\mathrm{C} 14 & 61.0(7) \\ \mathrm{C} 2-\mathrm{C} 3-\mathrm{C} 4-\mathrm{C} 9 & 167.5(5) & \mathrm{C} 7-\mathrm{C} 8-\mathrm{C} 13-\mathrm{C} 15 & -60.1(7) \\ \mathrm{C} 2-\mathrm{C} 3-\mathrm{C} 4-\mathrm{C} 5 & -58.7(6) & \mathrm{C} 9-\mathrm{C} 8-\mathrm{C} 13-\mathrm{C} 12 & -49.9(7) \\ \mathrm{C} 3-\mathrm{C} 4-\mathrm{C} 5-\mathrm{C} 6 & 169.8(5) & \mathrm{C} 9-\mathrm{C} 8-\mathrm{C} 13-\mathrm{C} 14 & -166.4(6) \\ \mathrm{C} 9-\mathrm{C} 4-\mathrm{C} 5-\mathrm{C} 6 & -55.9(6) & \mathrm{C} 9-\mathrm{C} 8-\mathrm{C} 13-\mathrm{C} 15 & 72.5(8) \\ \mathrm{C} 3-\mathrm{C} 4-\mathrm{C} 5-\mathrm{C} 16 & -65.7(7) & \mathrm{C} 4-\mathrm{C} 9-\mathrm{C} 10-\mathrm{C} 11 & -168.9(5) \\ \mathrm{C} 9-\mathrm{C} 4-\mathrm{C} 5-\mathrm{O} 1 & -168.4(4) & \mathrm{C} 8-\mathrm{C} 9-\mathrm{C} 10-\mathrm{C} 11 & -54.1(7) \\ \mathrm{C} 3-\mathrm{C} 4-\mathrm{C} 5-\mathrm{O} 1 & 57.3(6) & \mathrm{C} 20-\mathrm{C} 9-\mathrm{C} 10-\mathrm{C} 11 & 69.9(7) \\ \mathrm{C} 3-\mathrm{C} 4-\mathrm{C} 9-\mathrm{C} 20 & 60.6(7) & \mathrm{C} 9-\mathrm{C} 10-\mathrm{C} 11-\mathrm{O} 2 & -127.6(8) \\ \mathrm{C} 5-\mathrm{C} 4-\mathrm{C} 9-\mathrm{C} 8 & 56.3(6) & \mathrm{C} 9-\mathrm{C} 10-\mathrm{C} 11-\mathrm{C} 12 & 54.9(8) \\ \mathrm{C} 5-\mathrm{C} 4-\mathrm{C} 9-\mathrm{C} 10 & 171.6(5) & \mathrm{O} 2-\mathrm{C} 11-\mathrm{C} 12-\mathrm{C} 13 & 132.0(8) \\ \mathrm{C} 5-\mathrm{C} 4-\mathrm{C} 9-\mathrm{C} 20 & -70.7(6) & \mathrm{C} 10-\mathrm{C} 11-\mathrm{C} 12-\mathrm{C} 13 & -50.5(9) \\ \mathrm{C} 9-\mathrm{C} 4-\mathrm{C} 5-\mathrm{C} 16 & 68.6(6) & \mathrm{C} 11-\mathrm{C} 12-\mathrm{C} 13-\mathrm{C} 8 & 45.0(8) \\ \mathrm{C} 3-\mathrm{C} 4-\mathrm{C} 9-\mathrm{C} 8 & -172.5(5) & \mathrm{C} 11-\mathrm{C} 12-\mathrm{C} 13-\mathrm{C} 14 & 163.2(6) \\ \mathrm{C} 3-\mathrm{C} 4-\mathrm{C} 9-\mathrm{C} 10 & -57.2(6) & \mathrm{C} 11-\mathrm{C} 12-\mathrm{C} 13-\mathrm{C} 15 & -80.4(8) \\ \mathrm{O} 1-\mathrm{C} 5-\mathrm{C} 6-\mathrm{C} 7 & 168.9(5) & & \end{array}$

Hydrogen-bond geometry $\left(\AA,{ }^{\circ}\right)$

\begin{tabular}{lllll}
\hline$D-\mathrm{H} \cdots A$ & $D-\mathrm{H}$ & $\mathrm{H} \cdots A$ & $D \cdots A$ & $D-\mathrm{H} \cdots A$ \\
\hline $\mathrm{C} 18-\mathrm{H} 18 A \cdots \mathrm{O} 2^{\mathrm{i}}$ & 0.93 & 2.59 & $3.501(9)$ & 167 \\
\hline
\end{tabular}

Symmetry code: (i) $x-1 / 2,-y+3 / 2,-z+1$. 